\title{
Pratiques
}

Linguistique, littérature, didactique

$173-174 \mid 2017$

Le déjà-là dans l'écriture

\section{Planificateur ou formulateur? Mise en évidence de deux stratégies de rédaction à partir d'un support imagé chez des élèves de CE1 et de CM2}

Writing based on a visual source of images: evidence of two writing strategies in the intermediate and upper grades of elementary school

Denis Alamargot, Marie-France Morin et Elise Drijbooms

\section{OpenEdition}

\section{Journals}

Édition électronique

URL : http://journals.openedition.org/pratiques/3334

DOI : $10.4000 /$ pratiques.3334

ISSN : 2425-2042

Éditeur

Centre de recherche sur les médiations (CREM)

Référence électronique

Denis Alamargot, Marie-France Morin et Elise Drijbooms, « Planificateur ou formulateur? Mise en évidence de deux stratégies de rédaction à partir d'un support imagé chez des élèves de CE1 et de CM2 », Pratiques [En ligne], 173-174 | 2017, mis en ligne le 10 mars 2017, consulté le 20 avril 2019. URL : http://journals.openedition.org/pratiques/3334; DOI : 10.4000/pratiques.3334

Ce document a été généré automatiquement le 20 avril 2019

(c) Tous droits réservés 


\section{Planificateur ou formulateur? Mise en évidence de deux stratégies de rédaction à partir d'un support imagé chez des élèves de CE1 et de CM2}

Writing based on a visual source of images: evidence of two writing strategies in the intermediate and upper grades of elementary school

Denis Alamargot, Marie-France Morin et Elise Drijbooms

\section{Introduction}

1 Lire consiste à décoder une trace écrite pour accéder aux informations qui y sont transcrites. Dans le cadre scolaire, l'activité de lecture la plus communément considérée est celle de «lecture-compréhension», impliquant pour l'élève de décoder successivement les unités linguistiques d'un texte, tout en élaborant progressivement un modèle mental du contenu véhiculé. Lire ne se limite toutefois pas à cette seule activité de réception. L'élève peut être amené à lire un texte pour rechercher et extraire des informations, notamment dans le cas de la rédaction à partir d'une source documentaire (Alamargot, Chanquoy \& Chuy, 2005). De même, les supports de lecture sont variés. Audelà de la page écrite d'un livre ou d'un album, les activités de lecture peuvent être mobilisées à partir d'images ou encore de supports composites alliant texte et image, comme dans le cas de manuels et d'ouvrages illustrés. 


\subsection{Lire pour rédiger}

2 Lire pour rédiger est une activité particulièrement complexe, car elle suppose non seulement de décoder, comprendre, extraire les informations de la source documentaire, mais également de planifier et de formuler un texte original à partir de ces informations extraites. Plusieurs variantes peuvent être considérées : poursuivre une amorce de texte (un incipit); synthétiser plusieurs documents; résumer un texte ou encore décrire un support imagé.

3 Sur le plan pédagogique, il est généralement considéré qu'un support imagé (c.-à-d. illustrations d'un album, schémas dans un manuel, etc.) représente une aide à la compréhension de texte, car facilitant l'établissement du modèle de situation et la compréhension des relations entre les évènements (Glenberg \& Langston, 1992; Oostendorp \& Goldman, 1999). De même, dans le cas de la rédaction, l'on peut penser que composer un texte à partir d'une source imagée (c.-à-d. décrire une image, des figures géométriques, raconter une histoire à partir de vignettes, etc.) facilite le processus de planification en délimitant et alimentant le contenu du texte (Alamargot et al., 2011; Dansac \& Alamargot, 1999). Un examen des travaux de recherche menés sur ces questions module toutefois cette vision favorable. D'une part, le support imagé n'est pas toujours garant de facilitation en lecture-compréhension. Il peut même avoir des effets négatifs dès lors que l'image ne peut être aisément analysée ou reliée au texte correspondant (Graesser, León \& Otero, 2002). D'autre part, si l'influence du support imagé a été étudiée à différentes reprises dans le cas de la production orale (par exemple : Kern, 1997, 2002 ; Kern \& Raffara, 2012), la production écrite à partir d'images, quant à elle, n'a fait l'objet que d'un nombre limité de travaux, en linguistique (Cogis \& Leblay, 2010) comme en psychologie cognitive (Alamargot et al., 2011 ; Dansac \& Alamargot, 1999 ; Holland, 1987).

\subsection{Contraintes cognitives induites par la source documentaire}

Les stratégies rédactionnelles développées par les élèves lorsqu'ils rédigent à partir d'un support imagé restent encore peu connues et la question des contraintes introduites par l'image à consulter se pose. Comme le notent S. Kern et A. Raffara (2012), «Les images peuvent servir d'appui dans l'organisation du récit. Mais la présence des images peut également contraindre le narrateur à suivre la structure du récit qu'il ne partage pas forcément. » (pp. 97-98). Sur le plan cognitif, imposer le contenu du texte peut conduire le rédacteur à interrompre fréquemment - et plus ou moins longuement - le flux de son écriture pour consulter le support imagé ou, au contraire, l'inciter à maintenir en mémoire les informations de l'image, pour privilégier la continuité de sa composition. Dans les deux cas, le traitement du support imagé va exiger des ressources cognitives qui peuvent faire défaut aux autres processus rédactionnels, comme la formulation linguistique. Cette compétition serait d'autant plus marquée chez le rédacteur novice chez qui le coût de la formulation, notamment des traitements orthographiques, est particulièrement élevé (Fayol \& Miret, 2005 ; Hayes, 2012 ; Olive et al., 2009 ; Pontart et al., 2013). Ainsi, le traitement du support imagé pourrait s'opérer au détriment de celui de l'orthographe ou, au contraire, le traitement de l'orthographe pourrait limiter celui du support imagé. 


\subsection{La présente étude}

$5 \quad$ L'objectif de cette étude est de mieux comprendre le système de contraintes qui pèse sur la rédaction à partir d'un support imagé et de décrire les stratégies rédactionnelles adoptées par des élèves plus ou moins experts face à ces contraintes. Pour ce faire, nous proposons d'évaluer et de comparer les traitements rédactionnels d'élèves de CE1 (novices dans l'activité rédactionnelle) à ceux d'élèves de CM2 (chez qui l'expertise rédactionnelle s'installe) lorsqu'ils rédigent un texte narratif à partir d'un même support imagé.

6 Le choix d'un type texte narratif s'appuie sur le développement des compétences rédactionnelles des élèves. Les enfants étant, dans notre culture, très tôt familiers avec le genre narratif (Fayol, 1985 ; Roth, 2000 ; Verhoeven \& Van Hell, 2008), il est ici possible de comparer les performances des deux niveaux scolaires sur la base de connaissances communes.

7 Pour évaluer finement les performances des élèves, nous proposons, à la suite de D. Alamargot et al. (2010), de procéder ici à des études de cas. Il s'agira de décrire les caractéristiques non seulement linguistiques des textes produits, mais également temporelles, en relevant les pauses et débits d'écriture, ainsi que la fréquence et la durée de consultation du support imagé au cours de la rédaction.

\section{Méthode}

\subsection{Participants}

8 Deux élèves de CE1 (8,2 ans; 8,1 ans) et deux élèves de CM2 (10,8 ans; 10,5 ans) ont participé à cette étude de cas, après tirage au sort dans deux classes d'une école de Poitiers (Vienne) et obtention du consentement éclairé et écrit des parents. Tous étaient de langue maternelle française, aucun n'avait de difficulté d'apprentissage signalée et n'avait redoublé. Les prénoms des élèves ont été changés pour respecter l'anonymat.

\subsection{Tâches et matériel}

9 Il a été demandé aux quatre élèves de rédiger une histoire en consultant librement une source imagée composée de huit vignettes décrivant, en huit évènements, les mésaventures de deux enfants jouant et cassant un chariot fabriqué par leur père (Figure 1). Cette image est extraite du TAK (Taaltoets Alle Kinderen), test diagnostique de la maîtrise du langage oral (Verhoeven \& Vermeer, 2001). 

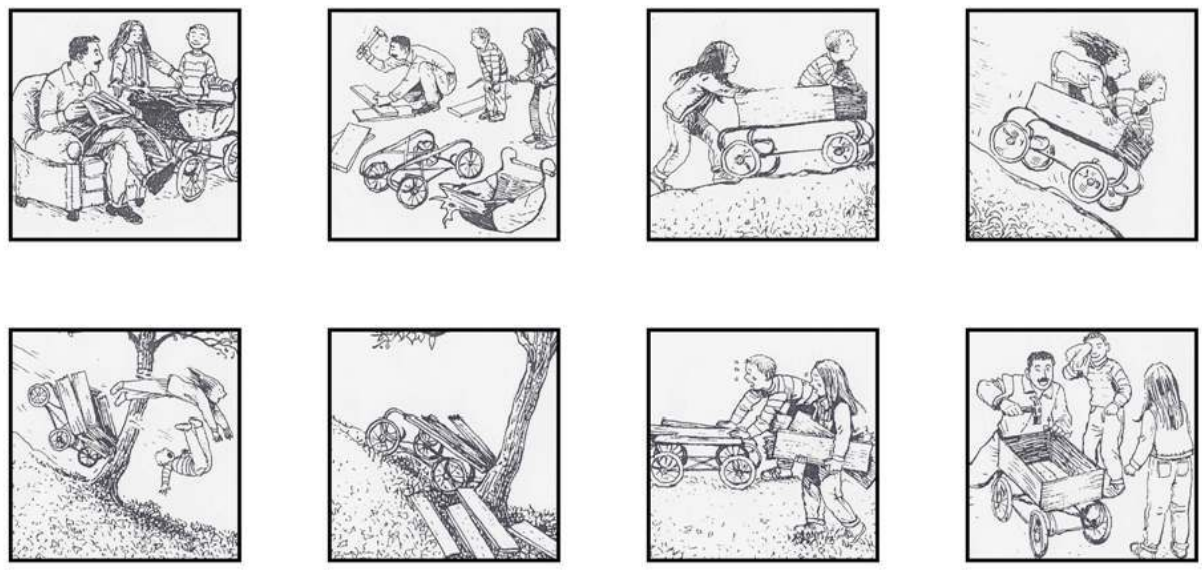

Pour enregistrer les paramètres temporels de l'écriture (c.-à-d. pauses, débits d'écriture et consultation de la source imagée), la tâche de rédaction a été effectuée sur une tablette-écran LCD (Wacom Cintiq 21UX) reliée à un ordinateur portable (Apple MacBook, piloté par le logiciel Eye and Pen ; Alamargot et al., 2006; Chesnet \& Alamargot, 2005 ; cf. Alamargot \& Morin, 2015 pour une discussion). Les élèves écrivaient à l'aide d'un stylet (Wacom Intuos 3 Grip Pen) directement sur l'écran de la tablette qui affichait la trace en temps réel. La source imagée, apparaissant à l'écran dès le début de la tâche de rédaction pour permettre aux élèves d'effectuer une consultation initiale, disparaissait avec le début de l'écriture. Durant la composition, les élèves pouvaient librement rappeler la source imagée à l'écran en appuyant la pointe du stylet sur une zone définie de la tablette. La source restait alors apparente le temps de l'appui. Ce dispositif permet d'enregistrer, en plus des pauses et débits d'écriture, la localisation dans le texte où la source imagée est consultée et combien de temps.

\subsection{Procédure}

Les passations individuelles se sont déroulées au sein de l'école. La rédaction, qui a duré entre 5 et 10 min selon les élèves, était effectuée sans contrainte temporelle. La consigne orale était la suivante : «Tu vas voir apparaître à l'écran une série d'images qui raconte une histoire avec un début et une fin. À toi de raconter cette histoire. Dès que tu commenceras à écrire, les images disparaitront. Mais tu peux les revoir aussi souvent et aussi longtemps que tu en as besoin en cliquant avec ton stylo sur le point vert. »

\subsection{Variables mesurées}

Les caractéristiques générales de la production du texte ont été évaluées par :

- la durée de composition (exprimée en min - excluant la consultation initiale de la source imagée et la pause finale),

- la durée de production moyenne d'un mot (exprimée en secondes/mots - c.-à-d. durée de composition divisée par le nombre de mots du texte). 
L'activité de planification du contenu du texte a été évaluée par :

- le nombre d'idées (propositions sémantiques, unités d'idées) contenues dans le texte et leur correspondance avec les 8 vignettes composant l'image,

- la durée de consultation initiale de l'image (exprimée en secondes - temps écoulé entre l'apparition de la source imagée à l'écran juste après l'énoncé des consignes et le début de l'écriture),

- le nombre de consultations ultérieures (effectuées durant la composition du texte),

- la durée totale de ces consultations ultérieures (exprimées en secondes).

L'activité de formulation a été évaluée par :

- le nombre de mots (chaîne de caractères séparés par un espace ou une apostrophe sauf dans le cas d'items lexicaux comme « aujourd'hui »),

- le nombre de phrases (conclues par un point),

- les pourcentages de connecteurs et de marques de ponctuation (en fonction du nombre de mots),

- le pourcentage de mots comportant au moins une erreur orthographique,

- le taux d'interruption de la production d'un mot par une pause longue. Une pause a été définie comme « longue » pour un élève donné dès lors qu'elle appartenait à l'ensemble des $25 \%$ des pauses les plus longues de cet élève; c'est-à-dire était élément du quartile supérieur de la distribution des pauses de l'élève (cf. Alamargot et al., 2010 ainsi que Prunty et al., 2014 qui utilisent le même critère). Sur le plan cognitif, l'on peut penser qu'une longue hésitation pendant l'écriture d'un mot traduit un problème majeur dans le traitement orthographique de ce mot.

\section{Résultats. Analyse des textes produits}

15 Les textes produits par les élèves sont transcrits ci-après. Les mots comportant au moins une erreur orthographique sont soulignés.

16 Clara (CE1) : Il était une fois une faimille povre qui contriusé une chario pour les enfants joue mais ils sont tombé du charrio et le chario sé case ils sont remonté et l'heure papa était unqué.

17 Victor (CE1) : En faite mon nistoi se passait dans une forèt à près $\underline{i l}$ on fabriqué une petite

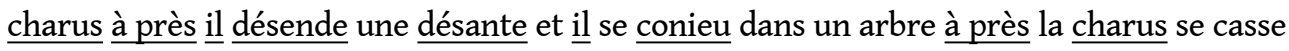

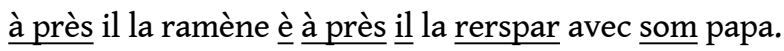

18

Élodie (CM2) : Les enfants demandent à leur père de construir une voiture en bois et le père se meit au travaille. Les enfants teste la voiture, ils vont vite! Mais maleresement, ils se prenent un arbre et la voiture est toute cassée. Ils ramenent la voiture à leur père et il se remit au travail

19

Justine (CM2) : Il était une fois une famille, avec le père, la fille et le fils. Le père aimait construire des choses, aujourd'hui il construisait un petit chariot pour ses enfants. Les

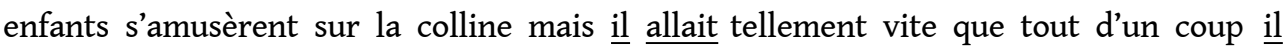
foncèrent dans un arbre et le chariot se cassa. Ils enmenèrent les restes du chariot à leur père, mais heureusement leur père ne se facha pas, vu qu'il aimait réparer des choses. Fin. 
Les performances générales et relatives à la planification et à la formulation du texte sont résumées dans le tableau 1.

Tableau 1. Caractéristiques des textes produits et performances rédactionnelles, en fonction du niveau scolaire et des élèves

\begin{tabular}{|c|c|c|c|c|c|}
\hline & \multirow{2}{*}{\begin{tabular}{|l} 
Niveau scolaire \\
Élève
\end{tabular}} & \multicolumn{2}{|l|}{ CE1 } & \multicolumn{2}{|l|}{$\mathrm{CM} 2$} \\
\hline & & Victor & Clara & Justine & Élodie \\
\hline \multirow{2}{*}{ Ensemble de la production } & Durée composition (min) & 9,55 & 5,32 & 9,65 & 7,25 \\
\hline & Durée production par mots (s/mots) & 11,24 & 8,44 & 7,15 & 8,05 \\
\hline \multirow{4}{*}{ Planification } & Nombre d'unités d'idées & 7 & 6 & 9 & 8 \\
\hline & Durée consultation initiale (s) & 13,48 & 78,24 & 31,11 & 66,53 \\
\hline & Nombre consultations suivantes & 14 & 2 & 13 & 3 \\
\hline & Durée consultations suivantes (s) & 73,50 & 13,93 & 79,55 & 39,76 \\
\hline \multirow{6}{*}{ Formulation } & Nombre de mots & 51 & 35 & 81 & 54 \\
\hline & Nombre de phrases & 1 & 1 & 5 & 4 \\
\hline & $\%$ de connecteurs $/$ mots & 9,80 & 5,71 & 6,17 & 7,40 \\
\hline & $\%$ de marques de ponctuation/mots & 1,96 & 5,71 & 8,10 & 8,26 \\
\hline & $\%$ de mots erronés & 37.25 & 40.00 & 7.40 & 11.11 \\
\hline & Taux de segmentation des mots & .23 & .31 & .10 & .18 \\
\hline
\end{tabular}

Deux phénomènes peuvent être décrits sur la base de cet ensemble de résultats. Le premier concerne les évolutions attendues des performances avec le niveau scolaire. Le second, sur lequel nous allons plus particulièrement nous focaliser, relève de l'existence de deux stratégies rédactionnelles qui, contrairement à nos attentes, semblent moins dépendre du niveau scolaire que de différences interindividuelles, quel que soit le niveau scolaire.

1) Concernant l'évolution avec le niveau scolaire, les résultats montrent une augmentation du nombre de mots et de phrases; des unités d'idées ainsi que des performances orthographiques. Sur le plan linguistique, la notion de phrases ne semble pas être encore maîtrisée par les deux élèves de CE1. Leurs textes ne sont constitués que d'une seule phrase, composée alors d'un nombre (trop) important de mots (Victor : 51 ; et Clara 35 mots par phrases). Les élèves de CM2, en revanche, segmentent clairement leurs textes en phrases (Justine: cinq ; Élodie : quatre phrases par texte en moyenne) au sein desquelles ils articulent les syntagmes par un plus grand nombre de marques de ponctuation (CE1 : Victor : 1,96; Clara : 5,71\%; CM2 : Justine : 8,10 ; Élodie : 8,26\%). En revanche, le pourcentage et l'emploi des connecteurs sont relativement similaires chez 
les CE1 (Victor : 9,80; Clara 5,71 \%) et les CM2 (Justine : 6,17 et Élodie : 7,40 \%) ; les quatre élèves en restant à l'utilisation du « et » générique, parfois complété par "mais » et/ou «après ». Sur le plan du contenu, le nombre d'idées produites par les élèves augmente sensiblement entre le CE1 (Victor : sept; Clara : six idées par texte) et le CM2 (Justine : neuf; Élodie: huit idées par texte). La répartition des idées selon les huit vignettes montre que les deux élèves de CE1 ne rendent pas compte de l'ensemble des évènements, notamment des vignettes 1 et 4 (pour Victor) et 3 et 4 (pour Clara) (c.-à-d. « les enfants demandent à leur père de construire un chariot »; « les enfants jouent avec le chariot »; "le chariot dévale la pente »). Enfin, une autre différence majeure entre les deux niveaux scolaires concerne les performances orthographiques. Le pourcentage de mots erronés relevé dans les textes est 3 à 4 fois plus faible chez les CM2 (Justine : 7,40 et Élodie: 11,11\%) que chez les CE1 (Victor: 37,25 et Clara: 40,00\%), ce qui est cohérent avec le développement attendu des compétences orthographiques (Manesse \& Cogis, 2007 ; Fayol, 2013). L'examen du taux de segmentation des mots par des pauses longues corrobore cette évolution. Le nombre de fois que la production d'un mot est interrompue par une pause longue est toujours plus important chez les CE1 (Victor : .23; Clara: .31) que chez les CM2 (Justine : .10 ; Élodie : .18).

2) Concernant les stratégies rédactionnelles adoptées pour planifier et formuler, l'analyse des résultats met en évidence d'importantes différences interindividuelles entre les élèves de même niveau scolaire. Chez les CE1, Victor met presque deux fois plus de temps pour rédiger que Clara (9,55 contre 5,32 $\mathrm{min}$ ), avec une durée de production par mots plus lente (11,24 contre $8,44 \mathrm{~s} / \mathrm{mots})$. Chez les CM2, Justine compose plus longtemps qu'Élodie ( 9,65 contre 7,25 $\mathrm{min}$ ), bien que produisant les mots plus rapidement $(7,15$ contre $8,05 \mathrm{~s} /$ mots). Ces variations semblent trouver leur origine dans des modes contrastées de consultation du support imagé. La stratégie de Clara (CE1) et d'Élodie (CM2) consiste à consulter longuement les images avant de commencer à écrire (respectivement 78,24 et $66,53 \mathrm{~s}$ ), pour ne faire ensuite que deux à trois consultations (pour une durée totale, respectivement, de : 13,93 et 39,76 s). La stratégie de Victor (CE1) et Justine (CM2) est radicalement opposée. Les deux élèves consultent peu de temps la source imagée avant de débuter l'écriture (respectivement 13,48 et 31,11 s) mais effectuent ensuite de nombreuses consultations (respectivement 13 et 12), venant fréquemment interrompre le flux de leur production (pour une durée totale, respectivement, de 73,50 et 79,55 s).

La question est de comprendre les raisons de ces différences interindividuelles qui semblent échapper au niveau scolaire et au développement de l'expertise rédactionnelle. Pour ce faire, nous avons analysé la localisation dans les textes des consultations de la source imagée et des pauses les plus longues, qui attestent de l'engagement d'une réflexion telle que l'écriture doit être interrompue. Les tableaux 2, 3, 4 et 5 présentent, pour chacun des élèves, la ventilation des caractères et mots produits entre deux consultations de la source imagée et/ou deux pauses longues. Nous décrivons après chaque tableau le détail des performances de chacun des élèves.

Tableau 2. Victor (CE1) : segmentation du texte produit en fonction des consultations de la source imagée et des pauses longues d'écriture

\begin{tabular}{|l|l|l|l|l|l|}
\hline Numéro & Texte & $\begin{array}{l}\text { Pauses } \\
(\mathrm{ms})\end{array}$ & Numéro & Texte & $\begin{array}{l}\text { Pauses } \\
(\mathrm{ms})\end{array}$ \\
\hline
\end{tabular}




\begin{tabular}{|c|c|c|c|c|c|}
\hline 1 & Consultation initiale & 13476 & 31 & (rature) & 3779 \\
\hline 2 & En $\mathbf{f}$ & 1668 & 32 & (rature) désante & 11315 \\
\hline 3 & aite & 2301 & 33 & Consultation & 5227 \\
\hline 4 & Consultation & 4092 & 34 & et & 1588 \\
\hline 5 & mon (rature) & 1723 & 35 & il se & 1716 \\
\hline 6 & nistoi & 2296 & 36 & conieu & 2596 \\
\hline 7 & Consultation & 3615 & 37 & Consultation & 4902 \\
\hline 8 & se pas & 1690 & 38 & A pres (rature) & 3140 \\
\hline 9 & sait & 2374 & 39 & Consultation & 2364 \\
\hline 10 & dans & 3200 & 40 & (rature) & 8095 \\
\hline 11 & Consultation & 464 & 41 & la charu & 2159 \\
\hline 12 & une & 2111 & 42 & $\mathrm{~s}$ & 1480 \\
\hline 13 & forèt & 3539 & 43 & Consultation & 5803 \\
\hline 14 & Consultation & 4211 & 44 & Se casse & \\
\hline 15 & $\mathbf{a}$ & 1796 & 45 & Consultation & 10667 \\
\hline 16 & près & 1543 & 46 & à $\mathbf{p}$ & 2024 \\
\hline 17 & il on & 1800 & 47 & rès & 1392 \\
\hline 18 & f & 1524 & 48 & il la ramè & 2215 \\
\hline 19 & abri & 13342 & 49 & ne & 1352 \\
\hline 20 & qué & 2144 & 50 & Consultation & 1903 \\
\hline 21 & Consultation & 8763 & 51 & è & 1888 \\
\hline 22 & une petite & 1455 & 52 & à pe & 1528 \\
\hline 23 & Consultation & 6915 & 53 & (ajout « r ») & 1640 \\
\hline 24 & charus & 3308 & 54 & $\mathrm{~s}$ il & 3259 \\
\hline 25 & Consultation & 10699 & 55 & la & 3079 \\
\hline 26 & à & 1727 & 56 & rers & 1600 \\
\hline 27 & (ajout d'un accent) & 2452 & 57 & par & 4332 \\
\hline
\end{tabular}




\begin{tabular}{|l|l|l|l|l|l|}
\hline 28 & près & 2439 & 58 & avec s & 2328 \\
\hline 29 & à près il désende une & 2891 & 59 & om & 1920 \\
\hline 30 & (correction d'un s) & 3479 & 60 & papa. & 1668 \\
\hline
\end{tabular}

L'examen du protocole de Victor (CE1) conduit à penser qu'il accorde une place importante à la formulation, priorité attestée par un pourcentage d'erreurs orthographiques et un taux de segmentation de mots moins élevés que ceux de Clara (CE1), assortis d'un effort de correction des mots (nombreuses opérations d'ajouts tout au long du texte). Il est probable que cet effort porté sur la formulation mobilise une part importante de ressources cognitives au détriment de la planification du contenu. Victor doit alors consulter plus fréquemment la source imagée pour élaborer, partie par partie, le contenu du texte. L'examen de la localisation des consultations corrobore cette interprétation. Sept des 13 consultations sont effectuées en dehors des transitions d'un épisode ; c'est-à-dire au cours même de la formulation de l'évènement (cf. consultations $\mathrm{n}$ $\left.{ }^{\circ} 4,7,11,21,23,39,43\right)$. Ce ne serait donc pas seulement le schéma narratif et ses épisodes qui guident les consultations de Victor, mais aussi la difficulté à maintenir en mémoire le contenu de la source imagée, sous la pression d'une formulation orthographique couteuse.

Tableau 3. Clara (CE1) : segmentation du texte produit en fonction des consultations de la source imagée et des pauses longues d'écriture

\begin{tabular}{|l|l|l|l|l|l|}
\hline Numéro & Texte & $\begin{array}{l}\text { Pauses } \\
(m s)\end{array}$ & Numéro & Texte & $\begin{array}{l}\text { Pauses } \\
(m s)\end{array}$ \\
\hline $\mathbf{1}$ & Consultation initiale & $\mathbf{7 8 2 3 7}$ & 23 & s sont & 7927 \\
\hline 2 & Il (rature) & 3004 & 24 & .(point)* & 1332 \\
\hline 3 & etait & 1723 & 25 & Q (majus)* & 6975 \\
\hline 4 & un & 1084 & 26 & tombé** & 2792 \\
\hline 5 & e fois & 1468 & 27 & du & 2608 \\
\hline 6 & une fai & 2144 & 28 & char & 5520 \\
\hline 7 & mille & 1344 & 29 & rio & 4383 \\
\hline 8 & povre* & 3603 & $\mathbf{3 0}$ & Consultation & $\mathbf{4 6 3 5}$ \\
\hline 9 & qui contri & 2456 & 31 & et & 2332 \\
\hline 10 & $\mathbf{u}$ & 1847 & 32 & le chario sé & 2059 \\
\hline 11 & se & 1148 & 33 & case & 4987 \\
\hline 12 & (accent de é) & $\mathbf{3 4}$ & Consultation & $\mathbf{9 2 9 1}$ \\
\hline
\end{tabular}




\begin{tabular}{|l|l|l|l|l|l|}
\hline 13 & une & 1000 & 35 & ils & 3976 \\
\hline 14 & cha & 2332 & 36 & Sont [s(pluriel)**] & 2292 \\
\hline 15 & rio & 1918 & 37 & remonté & 1663 \\
\hline 16 & pour & 3288 & 38 & et & 1145 \\
\hline 17 & les & 2394 & 39 & l'heure papa & 7019 \\
\hline 18 & enfants jou & 1312 & 40 & était & 1904 \\
\hline 19 & e & 3559 & 41 & un & 1123 \\
\hline 20 & mais & 2281 & 42 & q & 1248 \\
\hline 21 & i & 1432 & 43 & ué & 2596 \\
\hline 22 & $\mathbf{1}$ & 2368 & 44 &. & 2632 \\
\hline
\end{tabular}

Dans le cas de Clara (CE1), la compétition entre les processus de formulation et de planification ne semble pas être du même ordre que celle à l'œuvre chez Victor. Au niveau de la planification, la stratégie de consultation de Clara est à l'opposé de celle de Victor. Après une longue période d'observation initiale, elle effectue seulement deux courtes consultations, aux frontières des derniers épisodes. Elle a donc rédigé la plus grande partie de son texte à partir du contenu élaboré pendant la consultation initiale, en le maintenant en mémoire pendant une grande partie de la composition. Il est alors probable que cette priorité au maintien du contenu et à la planification réduise les ressources cognitives nécessaires à la formulation et au traitement orthographique. Cela conduirait à de nombreuses hésitations et erreurs, comme attesté par un pourcentage d'erreurs orthographiques ( $40 \%$ ) et un taux de segmentation des mots plus élevés que ceux de Victor.

Tableau 4. Élodie (CM2) : segmentation du texte produit en fonction des consultations de la source imagée et des pauses longues d'écriture

\begin{tabular}{|l|l|l|l|l|l|}
\hline Numéro & Texte & $\begin{array}{l}\text { Pauses } \\
(\mathrm{ms})\end{array}$ & Numéro & Texte & $\begin{array}{l}\text { Pauses } \\
(\mathrm{ms})\end{array}$ \\
\hline $\mathbf{1}$ & Consultation initiale & $\mathbf{6 6 5 3 4}$ & 30 &, ils & 9627 \\
\hline 2 & Les enfants demande & 1360 & 31 & vont & 1204 \\
\hline 3 & nt à leur & 2360 & 32 & vite! & 1407 \\
\hline 4 & père & 1516 & 33 & $($ point du !) & 5788 \\
\hline 5 & de c & 3284 & 34 & Mais & 1032 \\
\hline 6 & onstruir & 2055 & 35 & malere & 1687 \\
\hline
\end{tabular}




\begin{tabular}{|c|c|c|c|c|c|}
\hline 7 & $($ barre du $t)$ & 972 & 36 & sement & 1732 \\
\hline 8 & une (rature) & 2680 & 37 & , & 3522 \\
\hline 9 & (rature) & 1112 & 38 & il se & 5528 \\
\hline 10 & (rature) & 5295 & 39 & p & 1240 \\
\hline 11 & (rature) & 1924 & 40 & (ajour « s ») & 1027 \\
\hline 12 & (rature) & 2256 & 41 & renent & 2180 \\
\hline 13 & (rature) & 1616 & 42 & un & 1024 \\
\hline 14 & (rature) voi & 1620 & 43 & Consultation & 25293 \\
\hline 15 & $t$ & 1251 & 44 & arbre & 2560 \\
\hline 16 & ure & 1428 & 45 & et la voiture & 4648 \\
\hline 17 & en & 2658 & 46 & est toute & 1000 \\
\hline 18 & bois & 1353 & 47 & casse & 1036 \\
\hline 19 & Et le père se $\mathbf{m}$ (2 enjambements) & 10729 & 48 & (accent é) & 1116 \\
\hline 20 & $\mathrm{~m}\left(3^{\text {ème }}\right.$ enj $) \mathbf{e}$ & 2748 & 49 & e & 1547 \\
\hline 21 & it & 4104 & 50 & . & 2196 \\
\hline 22 & au travai & 2212 & 51 & Consultation & 9263 \\
\hline 23 & lle. & 1048 & 52 & Ils ramene & 1188 \\
\hline 24 & Consultation & 25293 & 53 & ent la voiture & 1116 \\
\hline 25 & Les & 1492 & 54 & à leur & 3014 \\
\hline 26 & (rature) & 1215 & 55 & père & 1024 \\
\hline 27 & (rature) enfant & 1004 & 56 & et il se remit au & 1100 \\
\hline 28 & teste la & 2572 & 57 & travail & 1136 \\
\hline 29 & voiture & 1004 & & & \\
\hline
\end{tabular}

La stratégie de consultation d'Élodie (CM2) s'apparente à celle de Clara (CE1). Elle ne consulte le support imagé qu'à la fin de la rédaction, et ce, aux frontières des évènements (la consultation $\mathrm{n}^{\circ} 43$ étant très proche de la frontière). Les performances liées à la formulation ont en partie le même profil que celles de Clara (CE1). Le taux de segmentation des mots reste élevé (.18), avec un pourcentage d'erreurs d'orthographe (11,11\%) important (comparativement à l'autre élève de CM2). Ainsi, Élodie (CM2), en 
donnant, comme Clara (CE1), la priorité à la planification, semble avoir elle aussi rencontré des problèmes de formulation.

Tableau 5. Justine (CM2) : segmentation du texte produit en fonction des consultations de la source imagée et des pauses longues d'écriture

\begin{tabular}{|c|c|c|c|c|c|}
\hline Numéro & Texte & $\begin{array}{l}\text { Pauses } \\
(m s)\end{array}$ & Numéro & Texte & $\begin{array}{l}\text { Pauses } \\
(m s)\end{array}$ \\
\hline 1 & Consultation initiale & 38979 & 34 & s'amusèrent sur la colline & 3208 \\
\hline 2 & Il était une fois & & 35 & mais il allait & 7340 \\
\hline 3 & Consultation & 2532 & 36 & tellement vite & 1176 \\
\hline 4 & une fami & 1400 & 37 & que tout d'un coup & 2907 \\
\hline 5 & lle & 1252 & 38 & Consultation & 4543 \\
\hline 6 & Consultation & 17634 & 39 & il fonce & 3168 \\
\hline 7 & , avec le père & 1584 & 40 & (accent de è) rent & 3508 \\
\hline 8 & , & 2816 & 41 & dans un & 15319 \\
\hline 9 & Consultation & 9967 & 42 & arbre & 1628 \\
\hline 10 & la fille et le fils & 1688 & 43 & Consultation & 6459 \\
\hline 11 & . & 1360 & 44 & et le chariot se cassa & 1424 \\
\hline 12 & Consultation & 8251 & 45 & . & 2187 \\
\hline 13 & Le père (rature) & 1700 & 46 & Consultation & 8099 \\
\hline 14 & (rature) & 1639 & 47 & Ils & 1804 \\
\hline 15 & (rature) aimait & 1388 & 48 & enmenèrent & 1852 \\
\hline 16 & construire & 1264 & 49 & les & 8315 \\
\hline 17 & des choses & 1692 & 50 & restes & 4171 \\
\hline 18 & , & 2024 & 51 & Consultation & 5032 \\
\hline 19 & Consultation & 3839 & 52 & du chariot & 1228 \\
\hline 20 & auj & 8699 & 53 & Consultation & 1468 \\
\hline 21 & ourd' (rature) & 2212 & 54 & à leur & 1176 \\
\hline 22 & (rature) hui il & 1972 & 55 & pere & 1528 \\
\hline
\end{tabular}




\begin{tabular}{|l|l|l|l|l|l|}
\hline 23 & construisait & 6183 & 56 & (accent de è) & 1408 \\
\hline 24 & Consultation & 4024 & 57 & ma & 5439 \\
\hline 25 & un & 2139 & 58 & is heureusement & 1976 \\
\hline 26 & petit & 1180 & 59 & leur père ne se facha pas & 3831 \\
\hline 27 & chariot & 1668 & $\mathbf{6 0}$ & Consultation & 2588 \\
\hline 28 & pour ses & 2984 & 61 &, & 3563 \\
\hline 29 & enfant & 1316 & 62 & vu qu'il ai & 1892 \\
\hline 30 & s & 4051 & 63 & (point sur i) m & 4083 \\
\hline 31 & Consultation & $\mathbf{5 1 1 1}$ & 64 & ait réparer & 1252 \\
\hline 32 & . & 4268 & 65 & des choses. Fin. & 1700 \\
\hline 33 & Les enfants & 2939 & & & \\
\hline
\end{tabular}

Justine, l'autre élève de CM2, développe un profil opposé. Ses consultations sont fréquentes et, comme Victor (CE1), en partie localisées entre les frontières d'évènements. Sur le plan de la formulation, le taux de segmentation des mots ainsi que le pourcentage de mots erronés sur le plan orthographique sont les plus faibles. Justine semble donc donner la priorité à la formulation et doit alors consulter le support imagé tout au long de la rédaction pour réussir à en planifier le contenu de façon très itérative.

\section{Discussion}

L'examen de ces quatre textes ainsi ventilés indique que deux stratégies de rédaction à partir d'un même support imagé semblent se dégager chez les quatre élèves : la priorité est donnée soit aux traitements orthographiques (mots erronées et segmentés moins nombreux) au détriment du maintien du contenu de l'histoire (nécessité alors d'opérer de courtes consultations tout au long de la composition); soit la priorité est donnée au maintien du contenu de l'histoire (longue planification initiale suivie de très peu de consultation) au détriment alors des traitements orthographiques (mots erronées et segmentés plus nombreux). La mise en évidence de deux stratégies différentes de rédaction chez des rédacteurs débutants confirme et précise les résultats de J.R. Hayes (2011), montrant l'existence précoce de différents modes de rédaction d'un texte expositif, résultant de différences interindividuelles et développementales. Les deux stratégies décrites ici dans le cadre d'un texte narratif ne sont pas sans rappeler également les deux grands modes de rédaction décrits par la critique génétique par les écrivains à programme - planificateur - et les écrivains à processus - formulateur (cf. Hay, 1993, 2002 ; Alamargot \& Lebrave, 2010 pour une synthèse).

Ce qui est marquant ici est la précocité (dès le CE1) de l'émergence de ces différences dans la gestion des traitements rédactionnels. L'engagement dans l'une ou l'autre de ces 
stratégies ne dépendrait pas de l'expertise des jeunes rédacteurs, car elles sont adoptées au CE1 comme au CM2. Chez ces élèves, il semble que ce soit une compétition entre les processus de planification et de formulation qui conduise à une mise œuvre temporelle différente des traitements rédactionnels durant la rédaction. La question du choix initial de l'une ou l'autre de ces stratégies reste toutefois posée. En effet, si la stratégie de consultation fréquente de la source imagée ne résultait que de la nécessité de réduire la surcharge cognitive due au traitement orthographique en cours d'écriture, alors les deux jeunes rédacteurs adoptant cette stratégie (c.-à-d. Victor et Justine) auraient tout à fait pu effectuer une pause initiale de planification longue comme les deux autres élèves, dans la mesure où la formulation n'a pas encore commencé à exercer des contraintes. Pourtant, la décision de ne pas planifier l'ensemble du contenu a été adoptée avant même d'éprouver les contraintes de la formulation, comme l'attestent les pauses initiales très courtes. Il semble donc que d'autres facteurs affectent également le choix d'une stratégie particulière, comme des différences interindividuelles sur le plan cognitif, possiblement liées à la capacité de la mémoire de travail (cf. pour une étude chez l'adulte : Alamargot et al., 2011) et/ou des connaissances métacognitives et métalinguistiques à propos de ses propres compétences et capacités en rédaction qui amènent très tôt à effectuer des choix dans la mise en œuvre des processus. Cette question reste à approfondir.

Un autre point de discussion relève de la généralisation possible de ces résultats à d'autres formes de rédaction. Nous avons choisi ici d'étudier un type particulier de narration réalisé à partir d'une source imagée. La question est de savoir dans quelle mesure les stratégies mises en évidence dans cette situation particulière sont également convoquées dans le cas d'une rédaction sans support (le contenu est alors récupéré et construit depuis la mémoire à long-terme) ou dont le support ne serait pas imagé mais textuel. Dans le cas de la poursuite d'un incipit, par exemple, le rédacteur doit non seulement s'appuyer sur le contenu sémantique du " pré-texte ", mais également intégrer et reprendre le vocabulaire et le niveau de langage alors imposés. Le rapport entre la planification et la formulation s'avérant alors très différent, il est possible que cela conduise à des stratégies rédactionnelles autres qu'il reste à découvrir.

Sur le plan méthodologique, le principe de consultation du support imagé piloté par la tablette (cliquer avec le style sur une zone déclenchant l'apparition des informations à l'écran - cf. Dansac \& Alamargot, 1999) constitue une méthode très intéressante pour étudier les modalités de consultation d'une source documentaire sans recourir à une oculomètre. De plus, cette méthode peut être mise en œuvre dans les classes de façon relativement simple. Une limitation toutefois porte sur la nécessité d'interrompre le flux de l'écriture pour faire apparaître les informations à consulter. Ce mode de consultation limite la possibilité de réaliser une lecture de la source documentaire parallèlement à l'écriture du texte, ce qui est la marque d'une expertise accrue comme ont pu le montrer D. Alamargot et al., (2010). Il serait sur ce point intéressant de comparer ces résultats avec ceux obtenus en connectant un oculomètre au logiciel Eye and Pen (Alamargot et al., 2006).

\section{Conclusion}

Cette étude de cas ouvre des perspectives de recherche quant à la présence et au rôle des différences interindividuelles dans les stratégies rédactionnelles. Les résultats obtenus auprès de ces quatre élèves montrent que la nature des stratégies déployées est 
probablement tributaire de la capacité du scripteur à gérer simultanément différents processus (ici planification, formulation) plutôt qu'à son niveau scolaire. La question de l'articulation du choix de la stratégie et des contraintes cognitives reste néanmoins à préciser, en lien avec les capacités mémorielles des élèves, leurs niveaux de connaissances en production écrite et les pratiques d'enseignement. Pour ce faire, il s'agira de mener également une recherche de plus grande ampleur, impliquant un nombre plus important et représentatif de participants permettant de généraliser ces premiers résultats. Le recours ici à une étude de cas n'en apparaît pas moins pertinent, à ce stade, pour décrire finement l'articulation entre le produit rédigé et la mise en œuvre temporelle des traitements. La ventilation des textes en fonction des pauses et consultations pour chaque sujet semble bien illustrer l'intérêt de la démarche pour baliser les recherches à venir mais également fournir aux enseignants des informations individualisées quant aux performances et stratégies de leurs élèves.

Finalement, sur le plan pédagogique, deux points doivent être considérés à partir de cette étude de cas. (i) Concernant l'activité de rédaction à partir d'un support imagé, il apparaît nécessaire de considérer la façon dont le support imagé est consulté par les élèves. En ce sens, les consignes données à l'élève devraient tenir compte de ses capacités, notamment orthographiques, et le diriger vers une consultation adaptée, favorisant plutôt la formulation chez les novices (c.-à-d. opérer des consultations fréquentes allégeant le maintien en mémoire). (ii) Comme l'explique la théorie capacitaire (Just \& Carpenter, 1992; McCutchen, 1996, 2011), les ressources cognitives limitées entraînent une compétition entre les processus non automatisés ou encore peu efficients. L'on constate, avec la description temporelle de l'activité rédactionnelle et de la segmentation des mots par des pauses, que le traitement de l'orthographe influence toujours l'activité rédactionnelle chez un élève de $\mathrm{CM} 2$, même lorsque la planification est supposée allégée par la stratégie de consultation du support imagé. La question de la maitrise effective de l'orthographe se pose ainsi toujours pour les élèves fréquentant la fin de l'école primaire, et ce, d'autant plus dans le cas d'un système orthographique comme le français qui est particulièrement inconsistant dans le sens de l'écriture.

\section{BIBLIOGRAPHIE}

ALAMARGOT, D., CAPOROSSI, G., CHESNET, D. \& ROS, C. (2011). « What Makes a Skilled Writer? Working Memory and Audience Awareness during Text Composition ». Learning and Individual Differences 21, p. 505-516.

ALAMARGOT, D., CHANQUOY, L. \& CHUY, M. (2005). «L'élaboration du contenu du texte : de la mémoire à long terme à l'environnement de la tâche ». Psychologie Française 50(3), p. 287-304.

ALAMARGOT, D., CHESNET, D., DANSAC, C. \& ROS, C. (2006). « Eye and Pen: a new device for studying reading during writing ». Behavior Research Methods 38(2), p. 287-299.

ALAMARGOT, D. \& LEBRAVE, J.-L. (2010). «The study of professional writing: A joint contribution from cognitive psychology and genetic criticism ». European Psychologist 15(1), p. 12-22. 
ALAMARGOT, D. \& MORIN, M.-F. (2015). « Does handwriting on a tablet screen affect students' graphomotor execution? A comparison between Grades 2 and 9 ». Human Movement Science 44, p. 32-41. En ligne : 10.1016/j.humov.2015.08.011.

AlAmARgot, D., PlAne, S., LAMBERT, E. \& CHESnET, D. (2010). « Using Eye and Pen Movements to Trace the Development of Writing Expertise: Case Studies of a Seventh, Ninth and Twelfth Grader, Graduate Student, and Professional Writer ». Reading and Writing 23(7), p. 853-888. En ligne :10.1007/s11145-009-9191-9.

CHESNET, D. \& ALAMARGOT, D. (2005). « Analyse en temps réel des activités oculaires et graphomotrices du scripteur : intérêt du dispositif “Eye and Pen" ». L'Année Psychologique 105(3), p. $477-520$.

COGIS, D. \& LEBLAY, C. (2010). « D'une version du texte à l'autre : aperçus sur la morphographie du nombre et sa révision en production verbale écrite ». Synergies Pays Scandinaves 5, p. 65-80.

DANSAC, C. \& ALAMARGOT, D. (1999). « Accessing referential information during text composition: when and why? » In : Torrance, M. \& Galbraith, D. (éds), Knowing what to write : Cognitive processes in the generation, selection and development of ideas during text production. Amsterdam : Amsterdam University Press, p. 79-97.

FAYOL, M. (1985). Le récit et sa construction. Paris : Delachaux \& Niestlé.

- (2013). L'acquisition de l'écrit. Paris : Presses universitaires de France.

FAYOL, M. \& MIRET, A. (2005). « Écrire, orthographier et rédiger des textes ». Psychologie Française 50, p. 391-402.

GLENBERG, A.M. \& LANGSTON, W.E. (1992). « Comprehension of illustrated text: Pictures help to build mental models ». Journal of Memory and Language 31(2), p. 129-151.

GRAESSER, A.C., LEÓN, J.A. \& OTERO, J. (éds) (2002). The Psychology of Science Text Comprehension. Mahwah, NJ : Erlbaum.

HAY, L. (éd.) (1993). Les manuscrits des écrivains. Paris : CNRS Éditions.

- (2002). La littérature des écrivains. Questions de critique génétique. Paris : Corti.

HAYES, J. R. (2012). « Evidence from language bursts, revision, and transcription form translation and its relation to other writing processes ». In : Fayol, M., Alamargot, D. \& Berninger, V. (éds), Translation of thought to written text while composing. New York: Psychology Press, p. 15-26. - (2011). « Kinds of Knowledge-Telling: Modeling Early Writing Development ». Journal of Writing Research 3, p. 73-92.

HOLLAND, V. M. (1987). Processes involded in writing procedural instructions. Graduate School, Pennsylvania State University, PhD Dissertation.

JUST, M. A. \& CARPENTER, P. A. (1992). « A capacity theory of comprehension: individual differences in working memory ». Psychological Review 99, p. 122-149.

KERN, S. (1997). Comment les enfants jonglent avec les contraintes communicationnelles, discursives et linguistiques dans la production d'une narration. Thèse de Doctorat des sciences du langage, Université Lumière - Lyon 2.

- (2002). «Contexte et acquisition du langage : un cas de narration ». Journal of French Language Studies 12(02), p. 181-201.

KERN, S. \& RAFFARA, A. (2012). «Effet du type de support imagé sur la production du récit chez l'enfant ». In : Delamotte, R. \& Akinci, M.A. (éds), Récits d'enfants : développement, genre, contexte. Rouen : Publications des universités de Rouen et du Havre., p. 97-115. 
MANESSE, D. \& COGIS, D. (2007). Orthographe : à qui la faute ? Paris : ESF éditeur.

MCCUTCHEN, D. (1996). « A capacity theory of writing: working memory in composition ». Educational Psychology Review 8, p. 299-325.

- (2011). « From novice to expert: implications of language skills and writing-relevant knowledge for memory during the development of writing skill ». Journal of Writing Research 3(1), p. 51-68.

OLIVE, T., FAVART, M., BEAUVAIS, C. \& BEAUVAIS, L. (2009). « Children's cognitive effort and fluency in writing: Effects of genre and of handwriting automatization ». Learning and Instruction 19, p. 299-308.

OOSTENDORP \& GOLDMAN (1999). The construction of mental representations during reading. Mahwah, NJ : Erlbaum.

PONTART, V., BIDET-ILDEI, C., LAMBERT, E., MORISSET, P., FLOURET, L. \& ALAMARGOT, D. (2013). «Influence of handwriting skills during spelling in primary and lower secondary grades ». Frontiers in Psychology 818(4), p. 1-9.

PRUNTY, M. M., BARNETT, A. L., WILMUT, K. \& PLUMB, M. S. (2014). « An examination of writing pauses in the handwriting of children with developmental coordination disorder ». Research in Developmental Disabilities 35, p. 2894-2905. En ligne : 10.1016/j.ridd.2014.07.033.

ROTH, F. P. (2000). « Narrative writing: Development and teaching with children with writing difficulties ». Topics in Language Disorders 20(4), p. 15-28.

VERHOEVEN, L. \& VAN HELL, J. G. (2008). « From knowledge representation to writing text: A developmental perspective ». Discourse Processes 45, p. 387-405.

VERHOEVEn, L. \& VERMEER, A. (2001). Taaltoets Alle Kinderen [TAK]. Arnhem : Cito groep.

VAN OOSTENDORP, H. E. \& GOLDMAN, S. R. (1999). The construction of mental representations during reading. Mahwah, NJ : Erlbaum.

\section{RÉSUMÉS}

L'objectif de cette recherche est de mieux comprendre le système de contraintes qui pèse sur la rédaction à partir d'un support imagé et de décrire les stratégies rédactionnelles adoptées par des élèves plus ou moins experts face à ces contraintes. Pour ce faire, nous avons évalué et comparé, par une étude de cas, les traitements rédactionnels de deux élèves de CE1 et de deux élèves de CM2 lorsqu'ils rédigeaient un texte narratif à partir d'un même support imagé (ici le " déjà-là »). L'analyse des caractéristiques linguistiques des textes produits a été complétée par une analyse du décours temporel de l'écriture, incluant les comportements de consultation du support imagé au cours de la rédaction. Les résultats mettent en évidence deux stratégies de rédaction à partir d'un même support imagé. L'une accorde la priorité aux traitements orthographiques, au détriment du maintien en mémoire du contenu de l'histoire. L'autre, à l'inverse, privilégie le maintien du contenu au détriment des traitements orthographiques. L'engagement dans l'une ou l'autre de ces stratégies ne semble pas dépendre de l'expertise des jeunes rédacteurs, car elles sont adoptées au CE1 comme au CM2. En conclusion, l'intérêt de ces résultats pour la mise en œuvre pédagogique d'activités rédactionnelles à partir de supports imagés est discuté.

The purpose of this study was to enhance our understanding of the constraints placed on writing, when composing a text based on a visual source of images. In particular, this study aimed to describe the compositional strategies adopted by children to deal with these constraints. To this 
aim, two children in the intermediate and two children in the upper grades of elementary school were asked to compose a narrative text based on images, after which their writing strategies were evaluated and compared. The results of this case study highlight two different strategies. Children using the first strategy highly focused on orthography, at the expense of the maintenance of the story content in memory. Children using the second strategy, by contrast, largely neglected orthographic errors, and prioritized the story content. The choice for one strategy over the other does not seem to depend on the expertise of the writers, as the two strategies occurred in both age groups. Educational implications for writing based on visual sources are discussed.

INDEX

Mots-clés : rédaction, développement, formulation, planification, stratégie, analyse_en_temps_réel

Keywords : text production, formulation, planification, strategies, real-time analysis, case study, elementary school

\section{AUTEURS}

\section{DENIS ALAMARGOT}

Université Paris-Est Créteil - France, CHArt, EA 4004, ESPÉ de l'Académie de Créteil, F-93526

\section{MARIE-FRANCE MORIN}

Université de Sherbrooke, CREALEC, Faculté d'éducation, Québec, Canada, CA-J1K 2R1

\section{ELISE DRIJBOOMS}

Radboud University Nijmegen, Behavioural Science Institute, Pays-Bas, NL-6525 HP 